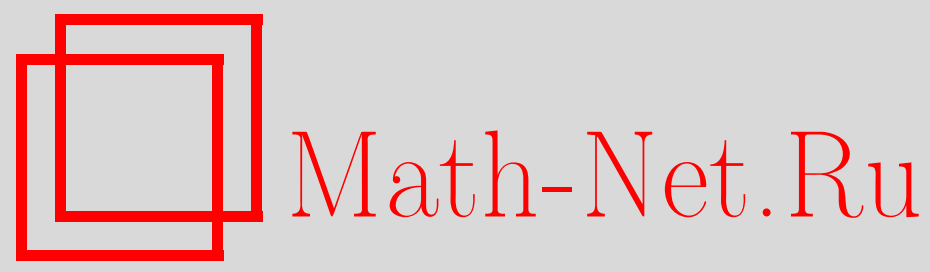

В. И. Малыхин, О подпространствах секвенциальных пространств, Матем. заметки, 1998, том 64, выпуск 3, 407-413

DOI: https://doi.org/10.4213/mzm1411

Использование Общероссийского математического портала Math-Net.Ru подразумевает, что вы прочитали и согласны с пользовательским соглашением http://www . mathnet.ru/rus/agreement

Параметры загрузки:

IP : 54.80 .73 .141

26 апреля 2023 г., 15:35:52

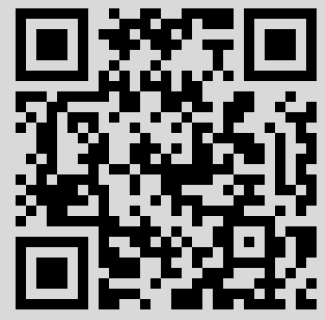




\title{
О ПОДПРОСТРАНСТВАХ СЕКВЕНЦИАЛЬНЫХ ПРОСТРАНСТВ
}

\section{В.И. Малыхин}

\begin{abstract}
Доказано, что пространство непрерывньх на обычном отрезке функций в топологии поточечной сходимости не субсеквенциально. В секвенциальных пространствах, удовлетворяющих определенным условиям, найдены плотные в себе подпространства без сходящихся последовательностей; такие подпространства найдены в некоторых секвенциальных компактах и полутопологических группах.
\end{abstract}

Библиограффия: 6 названий.

0. Введение. Подпространство секвенциального пространства называется субсеквенииальным. В данной заметке даются ответы на два вопроса, относящихся к этому классу пространств.

Вопрос 1 (А.В. Архангельский). Субсеквенциально ли $C_{p}(I)$-пространство непрерьвных на отрезке $I=[0,1]$ функций в топологии поточечной сходимости?

Ответ отрицателен, приводится пример не субсеквенциального подпространства пространства $C_{p}(I)$.

Вопрос 2 (Фольклор?). Должна ли быть сходящаяся последовательность в плотном в себе субсеквенциальном пространстве?

Вопрос интересен тем, что в самом секвенциальном пространстве (если оно не дискретно) сходящихся последовательностей всегда много, их достаточно для определения топологии пространства. (Одна из первых работ по секвенциальным пространствам (С.П. Франклин, 1965) так и названа (в переводе на русский) "Пространства, в которых последовательностей достаточно".)

Ответ и на этот вопрос отрицателен - доказывается теорема о наличии в секвенциальном пространстве, удовлетворяющем определенньм условиям, плотного в себе подпространства без сходящихся последовательностей; приводятся соответствующие примеры.

Напомним определение секвенииального пространства - это пространство, в котором множество замкнуто, если и только если оно содержит пределы всех своих сходящихся последовательностей. В теоретико-множественной топологии это один из важнейших классов пространств. Важное место этот класс занимает и в применениях, например, в теории функциональных пространств.

Субсеквенциальность, очевидно, наследуется подпространствами.

Приведем два важньх свойства субсеквенциальных пространств. 
ПРЕДЛОЖЕНИЕ 1. Пусть $X$ субсеквенииально, $A \subset X u a \in \bar{A} \backslash A$. Тогда а имеет счетную $\pi$-сеть из бесконечных подмнохсеств $A$, m.е. имеется счетное семейство бесконечных подмножеств $А$ такое, что любая окрестность а содержит некоторый әлемент этого семейства.

В сущности, это утверждение доказано Е. Г. Пыткеевым в [1].

ПРЕДЛОЖЕНИЕ 2. Хаусдорфово секвенциальное пространство и его подпространства имеют следующий вид тесноты: если а $\in \bar{A}$, то существует счетное $B \subset A, a \in \bar{B}$ и точки $B$ разделяются дизбюнктной системой окрестностей.

Доказательство этого предложения проходит стандартным образом по трансфинитной индукции (по индексу секвенциальности) и потому опускается.

1. Несубсеквенциальное подпространство $C_{p}(I)$. Рассмотрим следующеепространство $Z$, рассмотренное в [2]. Пусть $\left\{M_{k}: k \in \omega\right\}$ - дизъюнктное семейство конечных множеств, $\sup \left\{\left|M_{k}\right|: k \in \omega\right\}=\infty$. Пусть $M=\cup\left\{M_{k}: k \in \omega\right\}, * \notin M$ и $Z=\{*\} \cup M$. Пусть все точки $M$ изолированные, а типичная окрестность точки $*$ есть $\{*\} \cup(M \backslash L)$, где $\left|L \cap M_{k}\right| \leqslant m$ для всякого $k \in \omega$, а $m$ одно и то же для всех $k \in \omega$.

ПРЕДЛОЖЕНИЕ 3. Точка * не имеет счетной $\pi$-сети из бесконечных подмножеств $M$. Следовательно, пространство $Z$ не субсеквенииально.

ДокАЗАТЕЛЬСТво. Пусть $\left\{A_{i}: i \in \omega\right\}$ - какое-нибудь семейство бесконечньх подмножеств $M$. По индукции для всякого $k \in \omega$ найдем точку $x_{k} \in A_{k} \cap M_{i_{k}}$, причем все $i_{k}$ различны. Тогда $Z \backslash\left\{x_{k}: k \in \omega\right\}$ есть окрестность точки $*$, не содержащая ни одного множества $A_{i}$. Теперь на основании предложения 1 заключаем, что $Z$ не субсеквенциально.

ПРЕДЛОЖЕНИЕ 4. Пространство $C_{p}\left(D^{\omega}\right)$ содержит $Z$ как подпространство.

Для доказательства потребуется следующее утверждение.

КРИТЕРИЙ УСПЕНСКОГО (см., например, [3, с. 105]). Пусть все точки пространства $Z$, быть может, кроме точки *, изолированы в $Z$. Тогда подпространство $W=\left\{\chi_{F}: F \subset Z, * \notin \bar{F}\right\}$ пространства $D^{Z}$ является обвединением счетного семейства компактов в том и только том случае, если $Z$ гомеоморфно подпространству пространства $C_{p}(B)$ для некоторого компакта $B\left(\chi_{F}-\right.$ характеристическая функиия подмнохсества $F$ ).

Заметим сразу же, что из доказательства этого критерия в нашем случае ясно, что $B$ гомеоморфно $D^{\omega}$. Осталось убедиться, что в нашем случае $W$ есть объединение счетного семейства компактов.

Как следует из описания пространства $Z, W=\bigcup\left\{B_{m}: m \in \omega\right\}$, где $B_{m}=\left\{\chi_{F}\right.$ : $\left|F \cap M_{k}\right| \leqslant m$ для всякого $\left.k \in \omega\right\}$. Докажем, что $B_{m}$ замкнуто в $D^{Z}$. Действительно, если $t \in D^{Z} \backslash B_{m}$, то найдется $k_{0} \in \omega$ такое, что $\left|t^{-1}(1) \cap M_{k_{0}}\right|>m$. Однако, тогда $C=\left\{s \in D^{Z}: s^{-1}(1) \cap M_{k_{0}}=t^{-1}(1) \cap M_{k_{0}}\right\}$ открыто и $C \cap B_{m}=\varnothing$. Итак, $B_{m}$ замкнуто.

Приведем теперь несколько следствий.

Известно, что $C_{p}(B)$, где $B$ - компакт, есть пространство $\Phi$ реше-Урысона, если и только если $B$ - разреженный компакт (см., например, [3, с. 102]). Если же $B$ не разрежен, то он непрерывно отображается на обычный отрезок $I$, и тогда $C_{p}(I)$ есть подпространство $C_{p}(B)$. Так как $C_{p}\left(D^{\omega}\right) \subset C_{p}(I)$, из предложений 3,4 получаем 
СлЕДСТВИЕ 1. Пространство $C_{p}(B)$ не субсеквенииально, если $B$ - неразреженный компакт; в частности, $C_{p}(I)$ не субсеквенииально.

СлеДСТВИЕ 2. Пространство $C_{p}(X)$ не субсеквенииально, если $X$ - несчетное $A$-множество (в сепарабельном полном метризуемом пространстве).

Действительно, $X$ содержит $D^{\omega}$ как подпространство, отсюда следует, что $C_{p}\left(D^{\omega}\right) \subset$ $C_{p}(X)$.

ЗАмЕчаниЕ 1. В [4] доказано, что произведение $Z$ и веера $Ф$ реше-Урысона $V_{\text {c }}$ размера с имеют несчетную тесноту. Следовательно, и произведение $C_{p}(I) \times Z$ имеет несчетную тесноту. Тем самым, повторен соответствующий результат В. В. Успенского [5].

2. Нахождение подпространств без сходящихся последовательностей. Основньг инструментом в работе с такими подпространствами является понятие секвенциальной окрестности.

Все рассматриваемые последовательности предполагаются бесконечными.

Пусть $X$ - произвольное пространство. Для $A \subset X$ пусть $[A]^{1}=\{x: x$ есть предел сходящейся последовательности точек из $A\}$.

Секвенииальной окрестностью точки $x$ назьвается любое множество $W$ такое, что $x \notin[X \backslash W]^{1}$.

ПРЕДЛОЖЕНИЕ 5. Пусть $L-$ сходящаяся $\kappa$ точке последовательность, $W$ секвенииальная окрестность әтой точки. Тогда $|L \backslash W|<\aleph_{0}$.

В противном случае $L \backslash W$ есть сходящаяся к этой точке последовательность, что противоречит тому, что $W$ - ее секвенциальная окрестность.

Кратко можно сказать, что любая секвенциальная окрестность точки почти полностью содержит любую сходящуюся к этой точке последовательность.

ПРЕДЛОЖЕНИЕ 6. K точке сходится какая-нибудь последовательность, если и только если само это одноточечное множество не есть секвенциальная окрестность точки.

Действительно, если есть сходящаяся к этой точке последовательность, то почти вся эта последовательность содержится в любой секвенциальной окрестности; следовательно, любая секвенциальная окрестность бесконечна. Если же нет сходящихся последовательностей к точке $x$, то множество $\{x\}$ есть секвенциальная окрестность точки $x$.

ПРЕДЛОЖЕНИЕ 7. Секвенциальные окрестности точки в подпространстве пересечения с этим подпространством секвенциальных окрестностей этой точки во всем пространстве.

Просто проверяется.

Из этих предложений вытекает следующее

ПРЕДЛОЖЕНИЕ 8. Пусть $Y \subset X . B Y$ нет сходящихся последовательностей, если и только если для всякой точки $y \in Y$ найдется ее секвенциальная окрестность $W$ в $X$ такая, что $W \cap Y=\{y\}$.

Это предложение и дает способ нахождения и построения подпространств секвенциальных пространств без сходящихся последовательностей. 
ПРЕДЛОЖЕНИЕ 9. ПредПоложим, что у всякой точки $x$ :

(*) есть секвенииальная окрестность $W$ такая, что существует дизбюнктное семейство $\mathscr{V}$ окрестностей точек некоторого множсества $M$, так что $x \in \bar{M}$, но $(\cup \mathscr{V}) \cap W=\varnothing$.

Тогда существует плотное в себе подпространство без сходящихся последовательностей.

ДокАЗАТЕЛЬСТво. Пусть $x$ - произвольная точка. Тогда существуют секвенциальная окрестность $W_{x}$, множество $M_{x}$, точки которого имеют дизъюнктное семейство открытых окрестностей $\mathscr{V}_{x}=\left\{V_{y}: y \in M_{x}, V_{y} \ni y\right\}$, так что $x \in \bar{M}_{x}$, но $\left(\cup \mathscr{V}_{x}\right) \cap W_{x}=\varnothing$. Назовем точку $x$ точкой уровня 0 и обозначим множество $\{x\}$ через $K_{0}$. Назовем точки множества $M_{x}$ точками уровня 1 и обозначим множество $M_{x}$ через $K_{1}$.

Далее, для всякой $y \in K_{1}$ существуют своя секвенциальная окрестность $W_{y}$, множество $M_{y}$ и семейство $\mathscr{V}_{y}$ (см. вьше). Можно считать, $W_{y} \cup M_{y} \cup\left(\cup \mathscr{V}_{y}\right) \subset V_{y}$. Назовем точки множества $\bigcup\left\{M_{y}: y \in K_{1}\right\}$ точками уровня 2 и обозначим это множество через $K_{2}$; и т.д. После завершения описьваемого индуктивного процесса по всем натуральным числам получим для всякого $i \in \omega$ множество $K_{i}$ точек уровня $i$. Пусть $N$ - такое бесконечное подмножество $\omega$, что если $i \in N$, то $i-1 \notin N$. Пусть теперь $Y=\cup\left\{K_{i}: i \in N\right\}$. Докажем, что $Y$ - искомое подпространство. Так как множество точек какого-нибудь уровня содержит в своем замыкании все точки меньших уровней, $Y$ плотно в себе. А так как $W_{y} \cap Y=\{y\}$ для всякой точки $y \in Y$, в $Y$ нет сходящихся последовательностей.

ПРЕДЛОЖЕНИЕ 10. Предположим, что каждая точка хаусдорфова секвенииального пространства лежит на граниче некоторой своей замкнутой секвенциальной окрестности. Тогда существует плотное в себе подпространство без сходящихся последовательностей.

ДокАЗАтЕльство. Докажем, что вьполняется условие $(*)$ из предложения 9. В самом деле, пусть $x$ - произвольная точка. Тогда $x \in \overline{X \backslash W}$ для некоторой замкнутой секвенциальной окрестности $W$. Следовательно, на основании предложения 2 существует счетное $B \subset X \backslash W, x \in \bar{B}$ и точки $B$ разделяются дизъюнктной системой открытых окрестностей $\mathscr{V}$. Так как $B \cap W=\varnothing$, а $W$ замкнуто, можно считать, что $(\cup \mathscr{V}) \cap W=\varnothing$.

Заметим теперь, что вьполнение условия $(*)$ для точки регулярного секвенциального пространства в точности означает, что это - не точка Фреше-Урысона. Итак, справедлива

ТЕОрема 1. Если в регулярном секвенииальном пространстве каждая точка не есть точка Фреше-Урысона, то в нем существует плотное в себе подпространство без сходящихся последовательностей.

ТЕорема 2. В однородном регулярном секвенциальном не Фреше-Урысона пространстве существует плотное в себе подпространство без сходящихся последовательностей.

Таково, например, пространство свободной группы бесконечного метризуемого компакта.

Обратимся теперь к подпространствам компактов. 
ПРЕДЛОЖЕНИЕ 11. Пусть $Z=\{*\} \cup \omega$ - счетное бесконечное пространство без сходящихся последовательностей и с одной неизолированной точкой *. Тогда $Z^{\omega}$ содержит плотное в себе подпространство без сходящихся последовательностей.

ДокАЗАтЕЛЬСтво. Рассмотрим в $Z^{\omega}$ подпространство $X$, состоящее из точек, все координаты которых, кроме конечного числа, есть $*$ (так называемое $\sigma$-произведение с центром в точке $\bar{*}=(*, *, \ldots))$. Пусть $x-$ произвольная точка $X$. Тогда $x(n)=*$ для некоторого $n \in \omega$. Напомним, что $Z=\{*\} \cup \omega$. Для всякого $i \in \omega$ пусть $V_{i}=\left\{z \in Z^{\omega}: z(n)=i\right\}$ и $y_{i}$ - точка из $V_{i}$, на всех остальных (кроме $n$ ) координатах совпадающая с $x$. Нетрудно проверить, что для (произвольной) точки $x \in X$ условие (*) из предложения 9 вьполнено (множество $V_{i}$ есть дизъюнктные окрестности точек $y_{i}, X \backslash\left\{V_{i}: i \in \omega\right\}$ есть замкнутая секвенциальная окрестность точки $x$ ). Следовательно, согласно предложению 9 $X$ содержит плотное в себе подпространство без сходящихся последовательностей.

Напомним далее, что счетное произведение секвенциальных компактов секвенциально. Поэтому справедлива

ТЕОрема 3. Произведение счетного бесконечного числа секвенциальных не Фреше-Урысона компактов содержит плотное в себе подпространство без сходящихся последовательностей.

Действительно, каждьй из сомножителей имеет подпространство, гомеоморфное пространству $Z$ из предложения 11.

СлЕДСТВИЕ 3. Существует плотное в себе пространство без сходящихся последовательностей, являющееся плотным подпространством некоторого секвенииального компакта.

Это пространство найдем, используя теорему 3. Возьмем какой-нибудь компакт Франклина $F$. Это секвенциальный не $\Phi$ реше-Урысона компакт. Согласно теореме 3 в $F^{\omega}$ есть плотное в себе подпространство $Y$ без сходящихся последовательностей. Замькание $Y$ в $F^{\omega}$ есть искомьй секвенциальный компакт.

ЗАмЕчАниЕ 2. Этот секвенциальный компакт представляет собой секвенциальный компакт нового типа. Ранее не было примера секвенциального компакта без изолированных точек и с плотным множеством, в котором нет сходящихся последовательностей.

3. Построение секвенциальных пространств, содержащих плотные в себе подпространства без сходящихся последовательностей. Если в предыдущей части заметки мы находили плотные в себе и без сходящихся последовательностей подпространства уже известных пространств, то в этой части построим секвенциальные пространства, в которых есть плотные в себе подпространства без сходящихся последовательностей.

"Проколотой" секвенциальной окрестностью точки назовем секвенциальную окрестность этой точки, из которой сама точка удалена.

ПРЕДЛОЖЕНИЕ 12. Пусть у точек пространства $X$ существует дизбюнктное семейство "проколотых" секвенииальных окрестностей $\left\{W_{x}: x \in X, W_{x} \ni x\right\}$. Тогда существует плотное в себе подпространство без сходящихся последовательностей. 
ДокАЗАТЕЛЬСТво. Пусть $x$ - произвольная точка пространства. Положим $K_{0}=$ $\{x\}$. Если множество $K_{n}$ уже определено, то положим $K_{n+1}=\bigcup\left\{W_{x}: x \in K_{n}\right\}$. Построенные множества $K_{n}$ дизъюнктны. Кроме того, $K_{n} \subset \bar{K}_{n+1}$ для любого $n \in \omega$. Пусть $Y=\cup\left\{K_{2 n}: n \in \omega\right\}$. Тогда $Y$ плотно в себе. А поскольку $W_{y} \cap Y=\varnothing$ для любого $y \in Y$, в $Y$ нет сходящихся последовательностей.

ПРЕДЛОЖЕНИЕ 13. Пусть у точек счетного пространства существует дизбюнктное семейство "проколотых" секвенциальных окрестностей. Тогда существует плотное подпространство без сходящихся последовательностей.

ДокАЗАТЕЛьство. Занумеруем точки пространства $\left\{x_{n}: n \in \omega\right\}$. Несколько усложним определение множеств $K_{n}$ из предыдущего доказательства. Именно, на шаге $n+1$, если $x_{n+1}$ принадлежит множеству $\cup\left\{K_{i}: i \leqslant n\right\}$, то $K_{n+1}$ оставим прежним, а если не принадлежит, то включим $x_{n+1}$ в $K_{n+1}$. Далее можно убедиться, что $Y$ плотно во всем пространстве.

Построим, наконец, весьма "хорошее” секвенциальноепространство, распадающееся в сумму двух плотньх тоже “хороших" подпространств без сходящихся последовательностей каждое.

Пусть $\Omega$ - семейство конечных подмножеств какого-нибудь счетного бесконечного множества $\omega$. Групповой операцией “+” в $\Omega$ будет операция симметрической разности. С этой операцией $\Omega$ есть булева группа, т.е. группа с элементами только второго порядка. Такая группа абелева. Нуль этой группы есть пустое множество.

Пусть $\mathscr{F}$ - фильтр кофинитных подмножеств $\omega$. Он определяет на $\Omega$ топологию $\tau$ следуюшим образом: $V \in \tau$, если и только если для каждого $x \in V$ найдется $F \in \mathscr{F}$ такое, что $F+x \subset V$.

Заметим для дальнейшего, что для каждой точки $x$ семейство ее секвенциальных окрестностей в $(\Omega, \tau)$ есть семейство $\{F+x: F \in \mathscr{F}\}$.

ПРЕДЛОЖЕНИЕ 14. $(\Omega, \tau)$ есть полутопологическая группа, пространство которой регулярно и секвенииально.

Доказательство этого предложения не имеет отношения к теме заметки и опускается. Напомним только, что полутопологическая групnа - это группа с топологией, относительно которой все сдвиги и взятие обратного элемента есть гомеоморфизмы. Следовательно, как топологическое пространство оно в высшей степени однородно.

Пусть $Z=\{x \in \Omega:|x|$ четно $\}$. Тогда $Z$ - подгруппа $\Omega$, плотная в ней. Взаимоотношения группы $\Omega$, ее подгрупшы $Z$ суммирует

ПРЕДЛОЖЕНИЕ 15. C топологией подпространства из $(\Omega, \tau) \quad Z$ есть полутопологическая группа, пространство которой не содержит сходящихся последовательностей (но, подчеркнем, субсеквенииально).

Сходящихся последовательностей нет потому, что для всякой точки $x \in Z$ найдется элемент $F$ фильтра $\mathscr{F}$ такой, что $(F+x) \cap Z=\{x\}$.

Пространство $(\Omega, \tau)$ интересно еще и тем, что оно есть пространство со слабой первой аксиомой счетности. Слабая первая аксиома счетности - это некоторое усиление секвенциальности.

Подытожим сказанное следующим утверждением. 
СлЕДСтвИЕ 4. Существует счетная полутопологическая группа, пространство которой регулярно и со слабой первой аксиомой счетности; в әтой әруппе есть плотная подгруппа, также являющаяся полутопологической группой; пространство этой подгруппы регулярно, субсеквенциально и не имеет сходящихся последовательностей ( и не имеет слабой первой аксиомы счетности).

Отметим, что дополнение $\Omega \backslash Z$ также плотно в $\Omega$ и не имеет сходящихся последовательностей.

Напомним, что группа со слабой первой аксиомой счетности метризуема [6].

Мы не можем получить больше, чем следствие 4 , ибо справедлива

ТЕОрема 4. Подгруппа секвенциальной группь или дискретна (и, значит, замкнута и секвенциальна), или содержит сходящуюся последовательность.

ДокАзАТЕЛЬСтво. Если подгруппа не дискретна, то она замкнута или нет. Если она замкнута, то она секвенциальна и, поскольку не дискретна, то содержит сходящуюся последовательность. Если не замкнута, то из нее к некоторой точке дополнения сходится последовательность $\left\{x_{i}: i \in \omega\right\}$. Но тогда $\left\{x_{i} x_{i+1}^{-1}: i \in \omega\right\}$ есть последовательность, лежащая в рассматриваемой подгрупше и сходящаяся к единице группы.

Вопрос АВТоРА. Должна ли быть секвенциальной подгруппа секвенциальной группы?

О.В. Сипачёва изящно ответила отрицательно следующим образом.

Пусть $K$ - какой-нибудь секвенциальньй не пакт Ф ранк лина - см. вьше). В таком компакте есть несеквенциальное подпространство; обозначим его $X$. Свободная группа $F(K)$ компакта $K$ секвенциальна. Докажем, что подгрупа $\widetilde{X} \subset F(K)$, порожденная $X$, несеквенциальна. Действительно, эта подгруппа замкнуто содержит несеквенциальное подпространство $X$ и, значит, сама несеквенциальна.

Автор благодарен участникам семинара А. В. Архангельского в Московском университете за полезное обсуждение данной заметки.

\section{СПИСОК ЦИТИРОВАННОЙ ЛИТЕРАТУРЫ}

[1] Пыткеев Е. Г. О максимальной разложимости пространств // Тр. МИАН. 1983. Т. 154. C. 209-213.

[2] Malykhin V. I., Tironi G. Weakly FU-spaces // Quaderni Matematica, II Serie. Univ. di Trieste 1996. V. 386. P. 1-9.

[3] Архангельский А. В. Топологические пространства функций. М.: Изд-во МГУ, 1989.

[4] Bella A., Malykhin V. I. Around tight point // Topology Appl. 1997. V. 20. P. 1-8.

[5] Успенский В. В. О спектре частот функциональных пространств // Вестн. МГУ. Сер. 1. Матем., мех. 1982. №1. С. 31-35.

[6] Недев С., Чобан М. М. О метризуемости топологических групп // Вестн. МГУ. Сер. 1. Матем., мех. 1968. №6. С. 18-20.

Государственная академия управления им. С. Орджоникидзе

E-mail: matem@acman.msk.su 